\title{
A new characterization of computable functions
}

\author{
Apoloniusz Tyszka
}

\begin{abstract}
Let $E_{n}=\left\{x_{i}=1, x_{i}+x_{j}=x_{k}, x_{i} \cdot x_{j}=x_{k}: i, j, k \in\{1, \ldots, n\}\right\}$. We present two algorithms. The first accepts as input any computable function $f: \mathbb{N} \rightarrow \mathbb{N}$ and returns a positive integer $m(f)$ and a computable function $g$ which to each integer $n \geq m(f)$ assigns a system $S \subseteq E_{n}$ such that $S$ is satisfiable over integers and each integer tuple $\left(x_{1}, \ldots, x_{n}\right)$ that solves $S$ satisfies $x_{1}=f(n)$. The second accepts as input any computable function $f: \mathbb{N} \rightarrow \mathbb{N}$ and returns a positive integer $w(f)$ and a computable function $h$ which to each integer $n \geq w(f)$ assigns a system $S \subseteq E_{n}$ such that $S$ is satisfiable over non-negative integers and each tuple $\left(x_{1}, \ldots, x_{n}\right)$ of non-negative integers that solves $S$ satisfies $x_{1}=f(n)$.
\end{abstract}

Let

$$
E_{n}=\left\{x_{i}=1, x_{i}+x_{j}=x_{k}, x_{i} \cdot x_{j}=x_{k}: i, j, k \in\{1, \ldots, n\}\right\},
$$

and let $\mathcal{R} n g$ denote the class of all rings $\boldsymbol{K}$ that extend $\mathbb{Z}$. Th. Skolem proved that any Diophantine equation can be algorithmically transformed into an equivalent system of Diophantine equations of degree at most 2, see [6, pp. 2-3], [5, pp. 3-4], [1, pp. 386-387, proof of Theorem 1], and [3, pp. 262-263, proof of Theorem 7.5]. The following result strengthens Skolem's theorem.

Lemma ([7]). Let $D\left(x_{1}, \ldots, x_{p}\right) \in \mathbb{Z}\left[x_{1}, \ldots, x_{p}\right]$. Assume that $d_{i}=\operatorname{deg}\left(D, x_{i}\right) \geq 1$ for each $i \in\{1, \ldots, p\}$. We can compute a positive integer $n>p$ and a system $T \subseteq E_{n}$ which satisfies the following two conditions:

Key Words: computable function, Davis-Putnam-Robinson-Matiyasevich theorem.

2010 Mathematics Subject Classification: Primary 03D20; Secondary 11U09.

Received: October 2012

Revised:January 2013

Accepted: January 2013 
Condition 1. If $\boldsymbol{K} \in \mathcal{R} n g \cup\{\mathbb{N}, \mathbb{N} \backslash\{0\}\}$, then

$$
\begin{gathered}
\forall \tilde{x}_{1}, \ldots, \tilde{x}_{p} \in \boldsymbol{K}\left(D\left(\tilde{x}_{1}, \ldots, \tilde{x}_{p}\right)=0 \Longleftrightarrow\right. \\
\left.\exists \tilde{x}_{p+1}, \ldots, \tilde{x}_{n} \in \boldsymbol{K}\left(\tilde{x}_{1}, \ldots, \tilde{x}_{p}, \tilde{x}_{p+1}, \ldots, \tilde{x}_{n}\right) \text { solves } T\right)
\end{gathered}
$$

Condition 2. If $\boldsymbol{K} \in \mathcal{R} n g \cup\{\mathbb{N}, \mathbb{N} \backslash\{0\}\}$, then for each $\tilde{x}_{1}, \ldots, \tilde{x}_{p} \in \boldsymbol{K}$ with $D\left(\tilde{x}_{1}, \ldots, \tilde{x}_{p}\right)=0$, there exists a unique tuple $\left(\tilde{x}_{p+1}, \ldots, \tilde{x}_{n}\right) \in \boldsymbol{K}^{n-p}$ such that the tuple $\left(\tilde{x}_{1}, \ldots, \tilde{x}_{p}, \tilde{x}_{p+1}, \ldots, \tilde{x}_{n}\right)$ solves $T$.

Conditions 1 and 2 imply that for each $\boldsymbol{K} \in \mathcal{R} n \cup\{\mathbb{N}, \mathbb{N} \backslash\{0\}\}$, the equation $D\left(x_{1}, \ldots, x_{p}\right)=0$ and the system $T$ have the same number of solutions in $\boldsymbol{K}$.

For $\boldsymbol{K} \in \mathcal{R} n g$, the Lemma is proved in [8]. For concrete Diophantine equations, it is possible to find much smaller equivalent systems of equations of the forms $x_{i}=1$, $x_{i}+x_{j}=x_{k}, x_{i} \cdot x_{j}=x_{k}$, see [2].

The Davis-Putnam-Robinson-Matiyasevich theorem states that every recursively enumerable set $\mathcal{M} \subseteq \mathbb{N}^{n}$ has a Diophantine representation, that is

$$
\left(a_{1}, \ldots, a_{n}\right) \in \mathcal{M} \Longleftrightarrow \exists x_{1}, \ldots, x_{m} \in \mathbb{N} W\left(a_{1}, \ldots, a_{n}, x_{1}, \ldots, x_{m}\right)=0
$$

for some polynomial $W$ with integer coefficients, see [5] and [4]. The polynomial $W$ can be computed, if we know a Turing machine $M$ such that, for all $\left(a_{1}, \ldots, a_{n}\right) \in \mathbb{N}^{n}$, $M$ halts on $\left(a_{1}, \ldots, a_{n}\right)$ if and only if $\left(a_{1}, \ldots, a_{n}\right) \in \mathcal{M}$, see [5] and [4].

Theorem 1. There is an algorithm which accepts as input any computable function $f: \mathbb{N} \rightarrow \mathbb{N}$ and returns a positive integer $m(f)$ and a computable function $g$ which to each integer $n \geq m(f)$ assigns a system $S \subseteq E_{n}$ such that $S$ is satisfiable over integers and each integer tuple $\left(x_{1}, \ldots, x_{n}\right)$ that solves $S$ satisfies $x_{1}=f(n)$.

Proof. By the Davis-Putnam-Robinson-Matiyasevich theorem, the function $f$ has a Diophantine representation. It means that there is a polynomial $W\left(x_{1}, x_{2}, x_{3}, \ldots, x_{r}\right)$ with integer coefficients such that for each non-negative integers $x_{1}, x_{2}$,

$$
x_{1}=f\left(x_{2}\right) \Longleftrightarrow \exists x_{3}, \ldots, x_{r} \in \mathbb{N} W\left(x_{1}, x_{2}, x_{3}, \ldots, x_{r}\right)=0
$$

By the equivalence (E1) and Lagrange's four-square theorem, for any integers $x_{1}, x_{2}$, the conjunction $\left(x_{2} \geq 0\right) \wedge\left(x_{1}=f\left(x_{2}\right)\right)$ holds true if and only if there exist integers

$$
a, b, c, d, \alpha, \beta, \gamma, \delta, x_{3}, x_{3,1}, x_{3,2}, x_{3,3}, x_{3,4}, \ldots, x_{r}, x_{r, 1}, x_{r, 2}, x_{r, 3}, x_{r, 4}
$$

such that

$$
W^{2}\left(x_{1}, x_{2}, x_{3}, \ldots, x_{r}\right)+\left(x_{1}-a^{2}-b^{2}-c^{2}-d^{2}\right)^{2}+\left(x_{2}-\alpha^{2}-\beta^{2}-\gamma^{2}-\delta^{2}\right)^{2}+
$$




$$
\left(x_{3}-x_{3,1}^{2}-x_{3,2}^{2}-x_{3,3}^{2}-x_{3,4}^{2}\right)^{2}+\ldots+\left(x_{r}-x_{r, 1}^{2}-x_{r, 2}^{2}-x_{r, 3}^{2}-x_{r, 4}^{2}\right)^{2}=0
$$

By the Lemma for $\boldsymbol{K}=\mathbb{Z}$, there is an integer $s \geq 3$ such that for any integers $x_{1}, x_{2}$,

$$
\left(x_{2} \geq 0 \wedge x_{1}=f\left(x_{2}\right)\right) \Longleftrightarrow \exists x_{3}, \ldots, x_{s} \in \mathbb{Z} \Psi\left(x_{1}, x_{2}, x_{3}, \ldots, x_{s}\right)
$$

where the formula $\Psi\left(x_{1}, x_{2}, x_{3}, \ldots, x_{s}\right)$ is algorithmically determined as a conjunction of formulae of the forms:

$$
x_{i}=1, \quad x_{i}+x_{j}=x_{k}, \quad x_{i} \cdot x_{j}=x_{k}(i, j, k \in\{1, \ldots, s\})
$$

Let $m(f)=4+2 s$, and let $[\cdot]$ denote the integer part function. For each integer $n \geq m(f)$,

$$
n-\left[\frac{n}{2}\right]-2-s \geq m(f)-\left[\frac{m(f)}{2}\right]-2-s \geq m(f)-\frac{m(f)}{2}-2-s=0
$$

Let $S$ denote the following system

$$
\left\{\begin{aligned}
\text { all equations occurring in } \Psi\left(x_{1}, x_{2}, x_{3}, \ldots, x_{s}\right) & \\
n-\left[\frac{n}{2}\right]-2-s \text { equations of the form } z_{i}=1 & \\
t_{1} & =1 \\
t_{1}+t_{1} & =t_{2} \\
t_{2}+t_{1} & =t_{3} \\
& \cdots \\
t_{\left[\frac{n}{2}\right]-1}+t_{1} & =t_{\left[\frac{n}{2}\right]} \\
t_{\left[\frac{n}{2}\right]}+t_{\left[\frac{n}{2}\right]} & =w \\
w+y & =x_{2} \\
y+y & =y \text { (if } n \text { is even) } \\
y & =1 \text { (if } n \text { is odd) }
\end{aligned}\right.
$$

with $n$ variables. By the equivalence (E2), the system $S$ is satisfiable over integers. If an integer $n$-tuple $\left(x_{1}, x_{2}, x_{3}, \ldots, x_{s}, \ldots, w, y\right)$ solves $S$, then by the equivalence (E2),

$$
x_{1}=f\left(x_{2}\right)=f(w+y)=f\left(2 \cdot\left[\frac{n}{2}\right]+y\right)=f(n)
$$

A simpler proof, not using Lagrange's four-square theorem, suffices if we consider solutions in non-negative integers.

Theorem 2. There is an algorithm which accepts as input any computable function $f: \mathbb{N} \rightarrow \mathbb{N}$ and returns a positive integer $w(f)$ and a computable function $h$ which to each integer $n \geq w(f)$ assigns a system $S \subseteq E_{n}$ such that $S$ is satisfiable over non-negative integers and each tuple $\left(x_{1}, \ldots, x_{n}\right)$ of non-negative integers that solves $S$ satisfies $x_{1}=f(n)$. 
Proof. We omit the construction of $S$ because a similar construction is carried out in the proof of Theorem 1. The rest of the proof follows from the Lemma for $K=\mathbb{N}$.

For a function $f: \mathbb{N} \rightarrow \mathbb{N}$, let $\mathbb{Z}(f)$ denote the smallest $m \in\{1,2,3, \ldots\} \cup\{\infty\}$ such that for any integer $n \geq m$ there exists a system $S \subseteq E_{n}$ such that $S$ is satisfiable over integers and each integer tuple $\left(x_{1}, \ldots, x_{n}\right)$ that solves $S$ satisfies $x_{1}=f(n)$.

For a function $f: \mathbb{N} \rightarrow \mathbb{N}$, let $\mathbb{N}(f)$ denote the smallest $w \in\{1,2,3, \ldots\} \cup\{\infty\}$ such that for any integer $n \geq w$ there exists a system $S \subseteq E_{n}$ such that $S$ is satisfiable over non-negative integers and each tuple $\left(x_{1}, \ldots, x_{n}\right)$ of non-negative integers that solves $S$ satisfies $x_{1}=f(n)$.

The definition of $\mathbb{Z}(f)$ immediately implies that $\mathbb{Z}(f)=1$ for any $f: \mathbb{N} \rightarrow\{0,1\}$. By this and Theorem 1, we have the following.

Theorem 3. For any $f: \mathbb{N} \rightarrow \mathbb{N}$, if $f$ is computable, then $\mathbb{Z}(f)<\infty$, but not vice versa.

The analogous theorem holds for $\mathbb{N}(f)$.

\section{References}

[1] J. L. Britton, Integer solutions of systems of quadratic equations, Math. Proc. Cambridge Philos. Soc. 86 (1979), no. 3, 385-389.

[2] M. Cipu, Small solutions to systems of polynomial equations with integer coefficients, An. St. Univ. Ovidius Constanta 19 (2011), no. 2, 89-100, http://www. emis.de/journals/ASUO/mathematics/pdf23/Cipu.pdf, http://www . anstuocmath.ro/mathematics/pdf23/Cipu.pdf.

[3] M. Davis, Hilbert's tenth problem is unsolvable, Amer. Math. Monthly 80 (1973), no. 3, 233-269.

[4] L. B. Kuijer, Creating a diophantine description of a r.e. set and on the complexity of such a description, MSc thesis, Faculty of Mathematics and Natural Sciences, University of Groningen, 2010, http://irs.ub.rug.nl/dbi/4b87adf513823.

[5] Yu. Matiyasevich, Hilbert's tenth problem, MIT Press, Cambridge, MA, 1993.

[6] Th. Skolem, Diophantische Gleichungen, Julius Springer, Berlin, 1938.

[7] A. Tyszka, Conjecturally computable functions which unconditionally do not have any finite-fold Diophantine representation, Inform. Process. Lett. 113 (2013), no. 19-21, 719-722. 
[8] A. Tyszka, Does there exist an algorithm which to each Diophantine equation assigns an integer which is greater than the modulus of integer solutions, if these solutions form a finite set? Fund. Inform. 125(1): 95-99, 2013.

Apoloniusz Tyszka

University of Agriculture

Faculty of Production and Power Engineering

Balicka 116B, 30-149 Kraków, Poland

E-mail address: rttyszka@cyf-kr .edu.pl 
